\title{
The High-Tc Superconductive Gap Energy Evaluated by a Free Electron Laser Internal Photoemission
}

\author{
K. Nishi, K. Awazu ${ }^{*}$ and H. Horiike* \\ Toyohashi University of Technology, Toyohashi, Aichi 441-8580, Japan \\ Fax: 81-0532-44-6928, e-mail: nishi@eco.tut.ac.jp \\ *Osaka University, Suita, Osaka 565-0871, Japan
}

We investigated the high-temperature $\mathrm{YBa}_{2} \mathrm{Cu}_{3} \mathrm{O}_{7-\mathrm{x}}$ superconducting films by using a free electron laser (FEL). The method is a type of photoelectron spectroscopy called a free electron laser internal photoemission. The spectrum of the photocurrent induced by FEL was measured in the case of $15 \mathrm{~K}$ and $100 \mathrm{~K}$. We estimated the superconductive gap energy of $\mathrm{YBa}_{2} \mathrm{Cu}_{3} \mathrm{O}_{7-\mathrm{x}}$ by comparing the photocurrent spectrum of the superconductive state with that of non superconducive state.

Key words: Free electron laser; High-Tc superconductor

\section{INTRODUCTION}

In order to evaluate high-temperature cuprate supreconductors, various experimental methods have been devised to study the dynamical mechanism behind them [1]. Of these methods, the spectroscopic one such as photoelectron spectroscopy is a simple and direct method for evaluating the electronic structure of high-temperature superconductors [2]. We adopt a spectroscopic method using a free electron laser (FEL). This method is a type of photoelectron spectroscopy and is called a free electron laser internal photoemission. FEL has two main characteristics, wavelength tunability and ultrashort-pulse operation $(\sim 5 \mathrm{ps})$ with intense peak power $(\sim \mathrm{MW})$. The spectroscopy using FEL can be thus expected to have a high sensitivity of energy resolution. Since the superconductive gap energy of high-temperature superconductivity is identified as an order of $10 \mathrm{meV}$, the optical source of spectroscopy will be also required to have the similar photon energy (wavelength of $10 \sim 50 \mu \mathrm{m}$ ). In previous studies we reported that in $\mathrm{YBa}_{2} \mathrm{Cu}_{3} \mathrm{O}_{7-\mathrm{x}}$ superconducting films, photocurrent is induced by an infrared FEL $[3,4]$. In this paper we investigate the wavelength dependence of FEL-induced photocurrent in $\mathrm{YBa}_{2} \mathrm{Cu}_{3} \mathrm{O}_{7-\mathrm{x}}$ films.

\section{EXPERIMENTAL SETUP}

The detection of photocurrent, based on the principle of internal photoemission, is archived by amplifying and measuring the external photocurrent under optical pumping. A schematic for the FEL irradiation measurement is shown in Fig.1. The wavelength of FEL is $9.5 \mu \mathrm{m}$ and its beam consists of trains of 5 ps pulses (micropulses) with $45 \mathrm{~ns}$ separation. Moreover, the trains continues for about $20 \mu \mathrm{s}$ (macropulses) being repeated at $10 \mathrm{~Hz}$. The macropulse and micropulse of FEL are schematically shown in Fig. 2, respectively. The FEL is led to the $\mathrm{ZnSe}$ window of the cryostat through the mirrors and can be focused to the high-temperature superconductor sample in an optical cryostat using the convex lens. The irradiated area is about $1 \mathrm{~mm}^{2}$. The photocurrent signal was recorded on a digital oscilloscope triggered by the macropulse of the electron beam in the FEL apparatus. In order to detect the induced photocurrent more effectively, it was amplified by the low noise current amplifier. The sample $\left(\mathrm{YBa}_{2} \mathrm{Cu}_{3} \mathrm{O}_{7-\mathrm{x}}\right.$ film) was prepared on the $\mathrm{SrTiO}_{3}$ substrate by RF sputtering. Its critical temperature $\left(T_{c}\right)$ is about $80 \mathrm{~K}$. Two electrical contacts were made of deposited Ag. FEL was irradiated to the neighborhood of one electrode (electrical contact) on the $\mathrm{YBa}_{2} \mathrm{Cu}_{3} \mathrm{O}_{7-\mathrm{x}}$ film. FEL power was estimated by a pyroelectric joulemeter.

\section{RESULTS AND DISCUSSIONS}

Figure 3 shows the wavelength dependence of the photocurrent in the case of $15 \mathrm{~K}$. The wavelength is in the range of 9.5-12 $\mu \mathrm{m}$. Measurement was made in an unbias condition. Since FEL is a pulse laser (the macropulse width of $\sim 20 \mu$ s), the signal of the photocurrent becomes also pulse like. The starting point of the photocurrent signal corresponds to the irradiation of FEL. The photocurrent is normalized to a FEL power of $10 \mathrm{~mW}$. The sign of the current indicated is positive when the current flows from the $\mathrm{YBa}_{2} \mathrm{Cu}_{3} \mathrm{O}_{7-\mathrm{x}}$ to the $\mathrm{Ag}$ electrode. In the case of the wavelength of $9.5 \mu \mathrm{m}$, the sign of the photocurrent is positive at the

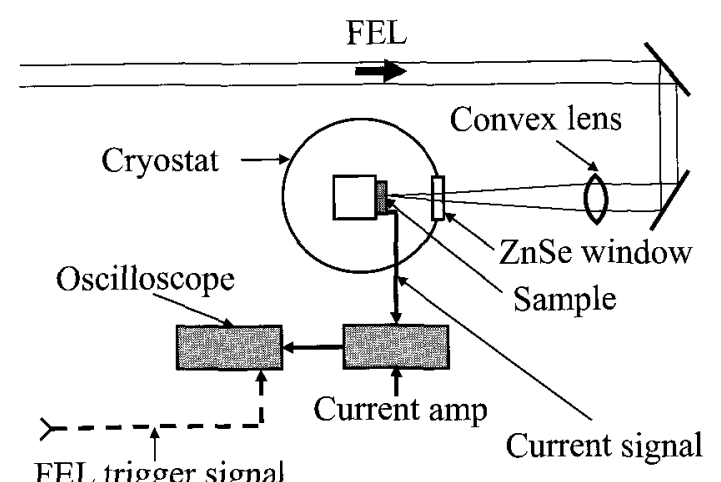

Fig. 1 Experimental setup. 


\section{macropulse}

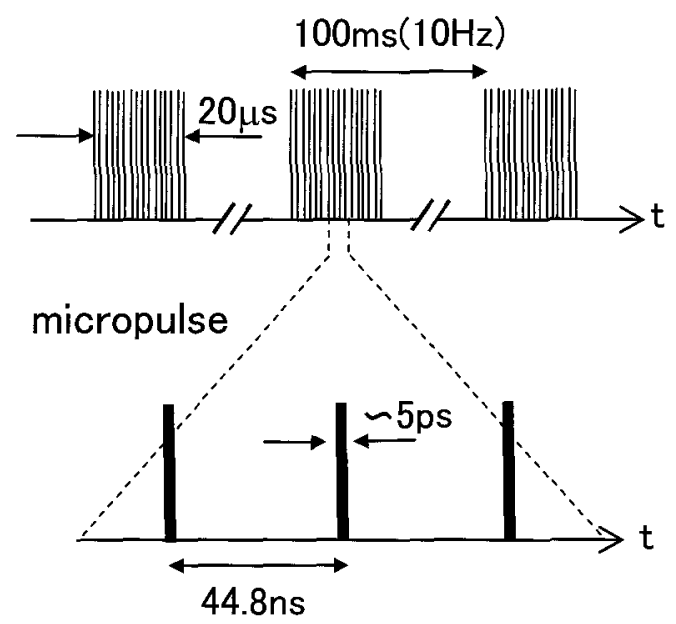

Fig. 2 Time structure of FEL.

starting point of the signal waveform, but changes approximately at the intermediate point of waveform. In the case of the wavelength of $9.5 \mu \mathrm{m}$, the sign of the photocurrent is positive at the starting point of the signal waveform, but changes approximately at the intermediate point of waveform. For increasing the wavelength, the point at which the sign of the photocurrent changes shifts to the signal starting point. Finally, in the case of $12 \mu \mathrm{m}$ the photocurrent is entirely negative. Figure 4 shows the photocurrent in the case of $100 \mathrm{~K}$. It is easily seen that the photocurrent decreases with increasing FEL wavelength. However, in the case of $100 \mathrm{~K}$, the sign of the photocurrent signal is positive in the range of $9.5-12 \mu \mathrm{m}$. We consider these results on the superconductor-metal interface model shown in Fig.5. First the waveform in Fig. 3 can be interpreted to consist of two current components with different response speed. It seems that the fast one corresponds to the current passing through the interface barrier and the slow one corresponds to the current reflected by the interface barrier. As shown in Fig. 6, the signal $S$ can be identified as the sum of two current components ( $\mathrm{A}$ and $B)$ which have different current directions. Here we obtained the component $\mathrm{A}$ by adjusting the component $\mathrm{B}$ as that the slow response (negative current) is negligible. On the other hand, in Fig.4 it seems that the current has only the fast component, though its response speed is somewhat different. This current will correspond to the carrier flow passing through the interface barrier.

In order to apply the analytic model to these experimental results, we consider the situation of a carrier which passes through the interface. This means that in order to evaluate the interface properties such as the energy of the interface barrier we only consider the contribution of the positive current indicated as $A$ in Fig.6. The photocurrent was averaged by taking the time integral of the current signal. Figure 6 shows the photon energy dependency of the averaging photocurrent in both cases of superconducting state (at $15 \mathrm{~K}$ ) and non-superconducting state (at $100 \mathrm{~K}$ ).

Now, we evaluate these data by using the following

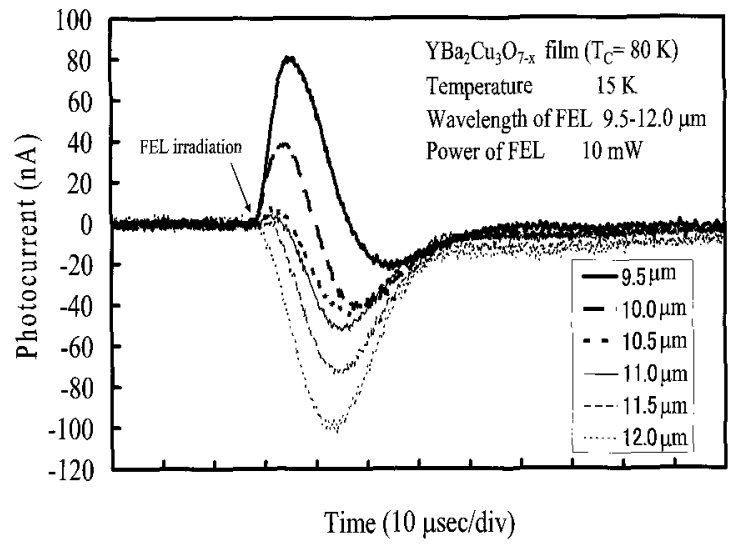

Fig.3 Photocurrent signals indicating the wavelength dependence at $15 \mathrm{~K}$ in the $\mathrm{YBa}_{2} \mathrm{Cu}_{3} \mathrm{O}_{7-\mathrm{x}}$.

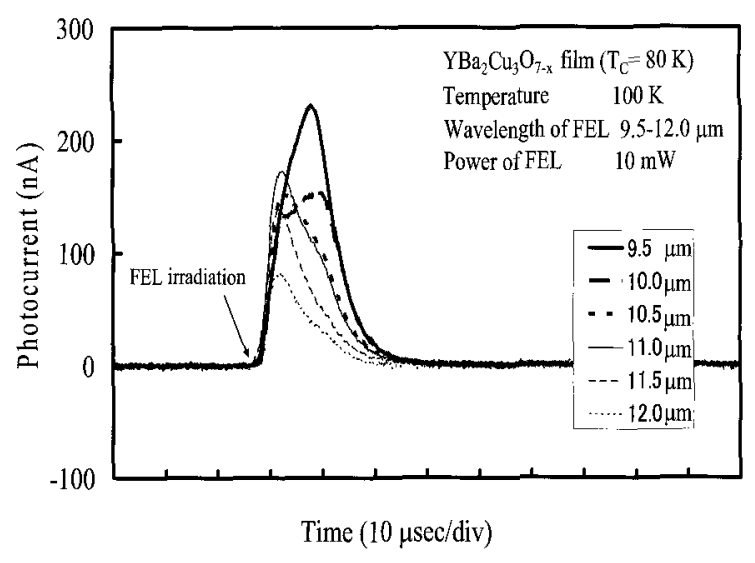

Fig.4 Photocurrent signals indicating the wavelength dependence at $100 \mathrm{~K}$ in the $\mathrm{YBa}_{2} \mathrm{Cu}_{3} \mathrm{O}_{7-\mathrm{x}}$.

formula for the photocurrent passing through the interface barrier [5].

$$
I=\frac{e W}{2\left(\overline{E_{p h}}\right)^{2}} \int_{1 \infty}^{E_{\infty}}\left\{1-\sqrt{\frac{\Delta}{E}}-\frac{1}{\alpha l}\left[\frac{1+\alpha l}{1+\alpha l \sqrt{\Delta / E}}\right]\right\} d E
$$

Here $e$ is the charge of electron, $W$ the FEL power, $E_{p h}$ the photon energy, $A$ the energy of the interface barrier, $\alpha$ the absorption coefficient, and $l$ is the mean free path of carriers. Using Eq. (1), we carried out a least mean square fitting. Fitting curves are reported in Fig. 7. As a result, we obtain two threshold energy of $\Delta_{n}=91 \mathrm{meV}$ and $\Delta_{\mathrm{s}}=105 \mathrm{meV}$. The latter value of $105 \mathrm{meV}$ is the threshold energy due to the excitation of carriers from the superconducting ground state to the interface barrier, as shown in Fig.6, while the value of $91 \mathrm{meV}$ is the threshold energy in the absence of superconducting gap (non superconducting state). We assumed that the interface barrier in non-superconducting state has a Schottky like behavior without temperature dependence. It is then suggested that the difference $\Delta_{\mathrm{s}}-\Delta_{\mathrm{n}}=14 \mathrm{meV}$ is corresponding to the superconducting gap. However, it is well known that the gap energy is anisotropic in the $\mathrm{CuO}_{2}$ (or a-b planes) of $\mathrm{YBa}_{2} \mathrm{Cu}_{3} \mathrm{O}_{7 \times \mathrm{x}}[6]$. Since FEL is 


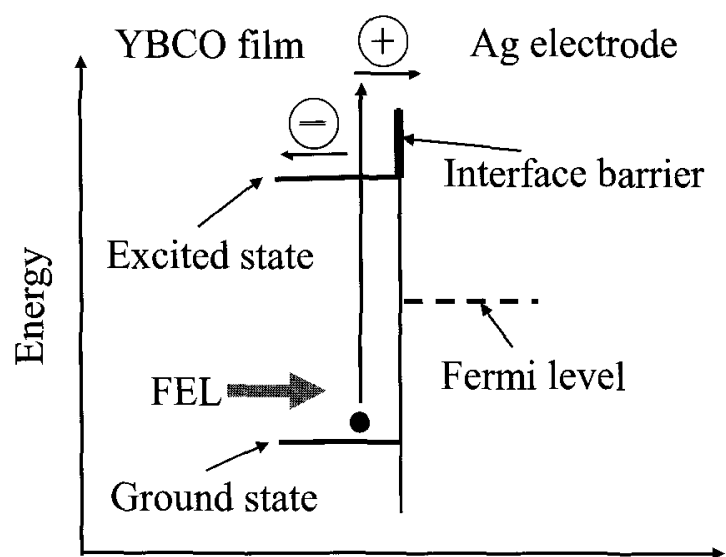

Fig. 5 A schematic energy diagram at the interface between $\mathrm{YBa}_{2} \mathrm{Cu}_{3} \mathrm{O}_{7-\mathrm{x}}$ film and $\mathrm{Ag}$ electrode.

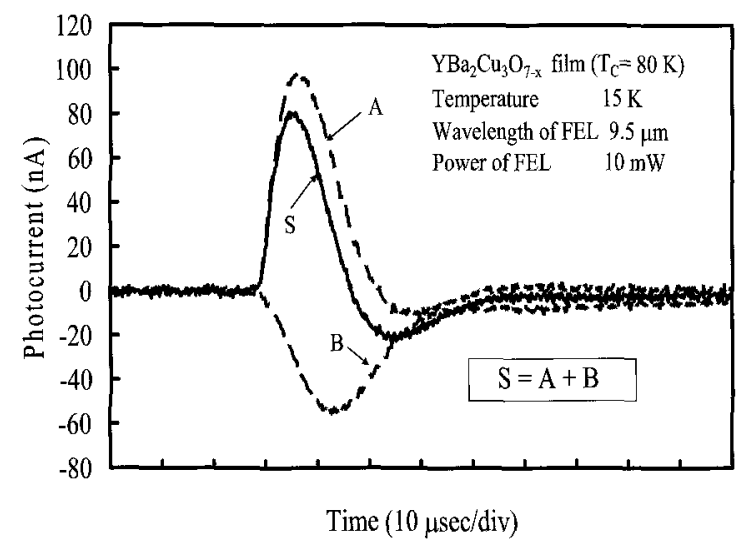

Fig.6 Structure of the photocurrent waveform. A total waveform is assumed to consist of two components.

irradiated perpendicular to $a-b$ planes of the $\mathrm{YBa}_{2} \mathrm{Cu}_{3} \mathrm{O}_{7-\mathrm{x}}$ film, the excited carriers will be emitted along both directions. It is then expected in our experiment that the photocurrent is an average level on anisotropic superconducting gap. Considering $d$-wave symmetry of $\mathrm{YBa}_{2} \mathrm{Cu}_{3} \mathrm{O}_{7-\mathrm{x}}$ superconductor, the average gap can be approximately estimated to be $56 \%$ of the maximum superconducting gap. We then obtain the value of $25 \mathrm{meV}(14 \mathrm{meV} / 0.56)$ as the maximum gap of $\mathrm{YBa}_{2} \mathrm{Cu}_{3} \mathrm{O}_{7-\mathrm{x}}$ film. This value is consistent with the gap of $\mathrm{YBa}_{2} \mathrm{Cu}_{3} \mathrm{O}_{7-x}$ estimated by angle-resolved photoemission spectroscopy (ARPES) [7].

This method has some advantages over the other photoelectron spectroscopy. One of them is that it is not so influenced by the surface state of samples. We can then investigate a variety of high $T_{\mathfrak{c}}$ superconductors. Other is the tunability of FEL which enables us to use an optical source with a variable wavelength. This will be powerful to carry out a precise measurement with high energy resolution. In addition, we can expect that utilizing the intense peak power of the FEL, it will become an effective method to search the nonequilibrium dynamics of the carriers in the high-temperature superconductor.

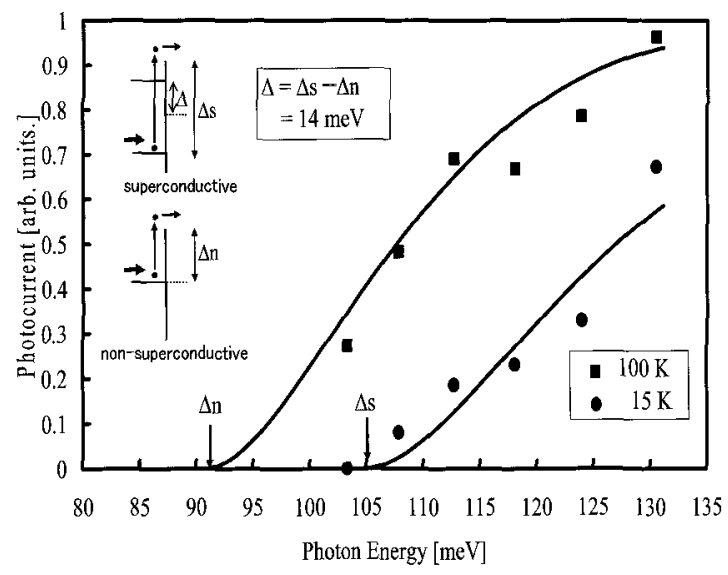

Fig.7 Spectrum of the photocurrent averaged by the time integral of the current signal.

\section{CONCLUSION}

We investigated the high-temperature $\mathrm{YBa}_{2} \mathrm{Cu}_{3} \mathrm{O}_{7-\mathrm{x}}$ superconducting films by using a free electron laser (FEL). We measured the FEL wavelength dependence of superconductive gap energy was estimated by analyzing the photocurrent spectrum at cases of $15 \mathrm{~K}$ and $100 \mathrm{~K}$. It is suggested that the maximum superconductive gap energy of $\mathrm{YBa}_{2} \mathrm{Cu}_{3} \mathrm{O}_{7-\mathrm{x}}$ is $\sim 25 \mathrm{meV}$. We intend to develop this analytic method in order to reveal the electronic structure of high temperature superconductors more precisely.

\section{References}

[1]C. C. Tsuei, J. R. Kirtley, Rev. Mod. Phys.,72, 969-1016(2000)

[2]J. M. Imer, F. Pstthey, B. Dardel, W.-D.

Schneider, Y. Baer, Y. Petroff, A. Zettl, Phys.Rev. Lett.,62,336-338(1989).

[3] K. Nishi, Y. Hatsukade, S. Tanaka, K. Awazu and H. Horiike, J. Magn. Magn. Mater.,310, 509 $-510(2007)$.

[4] K. Nishi, Y. Hatsukade, S. Tanaka, K. Awazu and H. Horiike, J. MRSJ., 32[1], 235-237(2007).

[5]C. N. Berglund, W. E. Spicer, Phys. Rev.,136, A1030A1044 (1964).

[6]Z. X. Shen et al., Phys. Rev. Lett., 70, 1553-1556 (1993).

[7] D. H. Lu et al., Phys. Rev. Lett.,86, 4370-4373

(2001).

(Recieved December 10, 2007 ; Accepted February 26, 2008) 